\title{
Kalman/Particle Filter for Integrated Navigation System
}

\author{
Zhun Jiao \\ Department of Aerial Instrument and Electric \\ Engineering \\ The First Aviation College of Air Force \\ Xin Yang, henan, China \\ Jiaozhun204@yahoo.com.cn
}

\author{
Rong Zhang \\ Air Force Engineering University \\ Xi'an, China \\ Zhangchubing_156@sohu.com
}

\begin{abstract}
Particle filter is introduced. Since the particle filter would bring hard computation, a new Kalman/Particle mixed filter used on SINS/GPS integrated navigation system was proposed. The new method divides the system into two submodels, one is linear, the other one is nonlinear, and then implement Kalman filter and particle filter separately. The simulation results show that their performance is almost equal, but the computation complexity of the Kalman/particle filter is much lower than traditional particle filter.
\end{abstract}

Keywords- integrated navigation system; particle filter; kalman/particle filter

\section{INTRODUCTION}

During warfare, the start-up time of navigation system needs to become less and less. The complicated environment, such as electronic interference, and instable GPS signal, is also needed to be overcome. To SINS/GPS integrated navigation system, when the SINS system starts, the azimuth error margin is big, and the non-linear factor during navigating can not be neglected. Furthermore, when the navigation equipment works in complicated environment, the noise model can not be made certain.

As the calculation speed is quickening, and the memory cost reducing, a new non-parameter modeling particle filter based on Bayes theorem is being attention gradually[1]. Particle Filter is put forward by Gordon and Salmond at 1993. The optimal solution of Physical model can be obtained via this method. And the filter can be applied to non-linear and non-Gaussian noise system. Much attention is also paid to this method in integrated navigation system[2,3]. To the ordinary Particle Filter algorithm, when the number of particle is tended to endless, particle distribution is constringency to real posterior probability density. When the number of particle is large, the calculation burden of ordinary particle filter algorithm aggravates. Therefore, when the number of state parameter is larger, the particle filter algorithm will be more complicated.

Considering the characteristic of particle filter algorithm and Kalman filter algorithm, a new Kalman/Particle mixed filter used on SINS/GPS integrated navigation system was proposed. The new method divides the system into two submodels, one is linear, the other one is nonlinear, and then implement Kalman filter and particle filter separately. This method can make the best use of dynamic properties of system. Further more, the state dimension of particle filter can be reduced, so that the dimension disaster would be avoided. With the large misalignment SINS error for model, we use the two filter method to simulate the SINS/GPS integrated navigation system. The simulation results show that, in the circumstances of the large misalignment angle, the Kalman/Particle mixed filter performs as well as the particle filter, but it is briefer in computation than particle filter.

\section{PARTICLE FILTER ALGORITHM}

\section{A. Non-linear and Non-Gaussian System Model}

Non-linear and non-Gaussian system can be described by the following dynamic state space model:

$$
\begin{gathered}
x_{k}=f_{k}\left(x_{k-1}, e_{k-1}\right) \\
y_{k}=h_{k}\left(x_{k}, v_{k}\right)
\end{gathered}
$$

$x_{k}\left(x_{k} \in R^{n_{x}}\right)$ is the state of system. $y_{k}\left(y_{k} \in R^{n_{y}}\right)$ is the measurement. $e^{k}$ and $v^{k}$ are system noise and measurement noise respectively. And they are separate. $f_{k}: R^{n_{x}} \times R^{n_{e}} \rightarrow R^{n_{x}}, h_{k}: R^{n_{x}} \times R^{n_{\nu}} \rightarrow R^{n_{y}}$, and they are bounded nonlinear maps. $n_{x}, n_{e}, n_{v}, n_{y}$ are the state vector, noise vector, measurement noise and measurement vector of the system respectively.

Based on the measurement data alignment, we use Bayesian estimation algorithm to estimate the conditional posterior probability distribution $p\left(x_{n} \mid y_{y}\right)$ of the state, especially the marginal probability distribution $p\left(x_{k} \mid y_{1: k}\right) \cdot x_{0: k}=\left\{x_{0}, x_{1}, \cdots x_{k}\right\}$ and $y_{1: k}=\left\{y_{1}, y_{2}, \cdots y_{k}\right\}$ are the initial marginal probability distributions. Here, we suppose that the initial marginal probability distribution function of the system state is $p\left(x_{0} \mid y_{0}\right)=p\left(x_{0}\right)$. We can get the posterior probability distribution of system state from Bayesian recursive formula and new measurement result $y_{k}$, like this:

$$
p\left(x_{k} \mid y_{1: k-1}\right)=\int p\left(x_{k} \mid x_{k-1}\right) p\left(x_{k-1} \mid y_{1: k-1}\right)
$$




$$
\begin{aligned}
p\left(x_{k} \mid y_{\mathrm{l}: k}\right) & =\left(p\left(y_{k} \mid x_{k}\right) p\left(x_{k} \mid y_{1: k}\right)\right) / p\left(y_{k} \mid y_{1: k-1}\right) \\
p\left(y_{k} \mid y_{1: k-1}\right) & =\int p\left(y_{k} \mid x_{k}\right) p\left(x_{k} \mid y_{1: k-1}\right) d x_{k}
\end{aligned}
$$

\section{B. Particle Filter Algorithm}

The PF is a recursive estimation method using Monte Carlo simulation within a Bayesian framework (Farina and Ristic, 2002; Arulampalam et a1., 2002). The central idea is to obtain the MMSE minimum mean-square error) of state from a set of random samples (particles) of state space to approximate the required probability density function (PDF). It is often impossible to directly sample from the posterior probability density. Thus, an importance proposal distribution, $q\left(x_{0: k} \mid z_{1: k}\right)$, with identical distribution, to the posterior distribution is introduced, where $z_{1: K}$ are all measurements from $\mathrm{t}=0$ to $\mathrm{t}=\mathrm{k}$. The bootstrap filter (Gordon et a1., 1993) simply takes the prior distribution as the proposal distribution by

$$
q\left(x_{k}^{i} \mid x_{0: k}^{i}, z_{1: k}\right)=p\left(x_{k}^{i} \mid x_{k-1}^{i}\right) \approx N\left(f\left(x_{k-1}^{i}\right), Q_{k-1}\right)
$$

in calculating the importance weights ${ }^{[4]}$. However, it would cause a larger error if there is little overlap between prior and the likelihood. To obtain more accurate proposal distribution within the particle filter flame, it may be used to update the mean and covariance of the Gaussian approximation to the state distribution given by

$$
q\left(x_{k}^{i} \mid x_{0: k-1}^{i}, z_{1: k}\right)=N\left(\hat{x}_{k}^{i} \mid x_{k-1}^{i}, P_{k \mid k}^{i}\right), i=1, \cdots, N \text {, }
$$

where $\mathrm{N}$ is the number of sampling particles ${ }^{[5]}$. So the $\mathrm{PF}$ can be derived (van der Merwe et a1., 2000). For completeness one cycle of the PF algorithm can be described in detail as follows:

(1) Initialization. Let $\left\{x_{0}^{x}\right\}_{t=1}^{N}$ be a set of particles sampled from the prior $p\left(x_{0}\right)$ at $\mathrm{k}=1$ and $\operatorname{set}_{w_{0}^{\prime}}=1 / N$.

(2)Update each particle $x_{k}^{\prime \prime} \sim p\left(x_{x} \mid x_{-1}^{\prime}\right), \quad \mathrm{i}=1, \cdots, \mathrm{N}$. Calculate the likelihood probability $p\left(z_{k} \mid x_{k}^{c}\right)$ of each particle. Update the weight $_{w_{k}^{r}}^{r}=p\left(z_{k} \mid x_{k}^{r}\right)$, and make it be normalized, like this ${ }^{w_{i}^{\prime}}=w_{i}^{\prime} / \sum_{i=1}^{n} w_{i}^{\prime}$ 。

(3)Let $\left\{x_{*}^{\prime}, w_{*}^{\prime}\right\}_{1}^{y}$ be Resample, and get the new

particles $\left\{x_{k}^{\prime}, w_{k}^{\prime}\right\}_{1}^{N}, \quad w_{i}^{k}=1 / N$;

(4) Let $\mathrm{k}=\mathrm{k}+1$, and return to the (2).

Wherever Times is specified, Times Roman or Times New Roman may be used. If neither is available on your word processor, please use the font closest in appearance to Times. Avoid using bit-mapped fonts if possible. True-Type 1 or Open Type fonts are preferred. Please embed symbol fonts, as well, for math, etc.

\section{KALMAN /PARTICLE FILTER ALGORITHM}

The dimensional state vector of dynamic model is divided into two sub-models, $\left[\begin{array}{ll}x_{k}^{\pi} & x_{k}^{n \pi}\end{array}\right]^{r}$ is linear, and $x_{k}^{1}, x_{k}^{n}$ is nonlinear. Then Kalman filter and particle filter are implemented separately.

The error model state vector of SINS can be described like this:

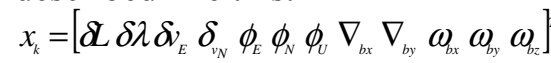

And then:

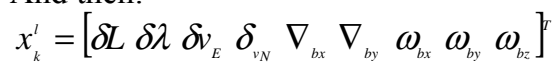

$x_{k}^{n}=\left[\phi_{E} \phi_{N} \phi_{U}\right]^{T}$

The error model and observation model equation of SINS is:

$x_{k+1}^{l}=F_{k}^{l}\left(x_{k}^{n}\right)+A_{k}^{l} x_{k}^{l}+w_{k}^{l}$

$x_{k+1}^{n}=F_{k}^{n}\left(x_{k}^{n}\right)+A_{k}^{n} x_{k}^{\prime}+w_{k}^{n}$

$z_{k}^{l}=H_{k} x_{k}^{l}+e_{k}^{l}$

where $F_{k}^{\prime}\left(x_{k}^{n}\right)=M\left(I-C_{p}^{n}\right) C_{b}^{p} \hat{f}^{b}, \quad F_{k}^{n}\left(x_{k}^{n}\right)=\left(I-C_{p}^{n}\right) \omega_{i n}^{n}, \quad A_{k}^{\prime}$ and $A_{k}^{n}$ are linear matrix. They can be calculated from Eq. (9). Where:

$$
\begin{aligned}
& M=\left[\begin{array}{c}
O_{2 \times 3} \\
100 \\
010 \\
O_{s \times 3}
\end{array}\right], \quad H_{k}=\left[\begin{array}{ll}
I_{4 \times 4} & O_{4 \times 8}
\end{array}\right], \quad \omega_{k} \in N\left(0, Q_{k}^{l}\right), \\
& \omega_{n} \in N\left(0, Q_{k}^{n}\right), \quad e_{k}^{l} \in N\left(0, R_{k}\right)
\end{aligned}
$$

After state decomposition, based on the Bayesian, we know that:

$$
\begin{aligned}
p\left(x_{k} \mid z_{I k}\right) & =p\left(x_{k}^{n}, x_{k}^{\prime} \mid z_{t k}\right) \\
& =\underbrace{p\left(x_{k}^{n} \mid x_{k}^{\prime}, z_{1 k}\right)}_{p F} \underbrace{p\left(x_{k}^{\prime} \mid z_{t k}\right)}_{k F}
\end{aligned}
$$

From (2.1), we find that:

$$
z_{k}^{n}=x_{k+1}^{l}+A_{k}^{\prime} x_{k}^{l}=F_{k}^{\prime}\left(x_{k}^{n}\right)+\omega_{k}^{l}
$$

where $p\left(x_{k}^{\prime} \mid z_{i k}\right)$ is estimated by Kalman filter, and $p\left(x_{k}^{n} \mid x_{k}^{1}, z_{1 \cdot k}\right)$ is estimated by Particle filter.

The Kalman/ Particle filter described in detail as follows:

(1) Initialization. We suppose that $x_{t \mid 0}^{\prime}, p_{t \mid}^{\prime}, p_{x_{0}^{n}}\left(x^{n}\right)$ are

known. To the linear model $\mathrm{Eq}(9)$ and $\mathrm{Eq}(10)$,

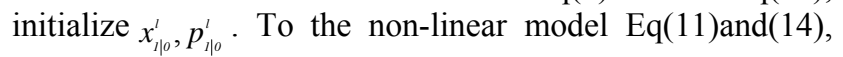
the particles $x_{0}^{n,(i)} \sim p_{x_{0}^{n}}\left(x_{0}^{n}\right)$ come into being random, and the number of the particles is $\mathrm{N}$. The weight is $q_{0}^{i}=1 / N$. Then $F_{o}^{\prime}\left(x_{0}^{n}\right)=M\left(1-C_{p}^{n}\right) C_{b}^{p} \hat{f}^{b}$, and $\mathrm{k}=1$ 。

(2) Update the measurement of Kalman filter:

$$
\begin{gathered}
x_{k}^{l}=x_{k \mid k-1}^{l}+F_{k-1}^{l} K_{K}\left(z_{k}^{l}-H_{k} x_{k \mid k-1}^{l}-H_{k} F_{k-1}^{l}\right)(15) \\
P_{K}=\left(1-K_{K} H_{k}\right) P_{k \mid k-1}
\end{gathered}
$$




$$
K_{\kappa}=p_{k \mid k-1} H_{k}^{T}\left(H_{k} P_{k \mid k-1} H_{k}^{T}+R_{k}\right)^{-1}
$$

(3) Update the time of Particle filter.

Bringing $\left\{x_{k-1}^{n,(i)}, q_{k-1}^{i}\right\}_{i=1}^{N}$ and $x_{k}^{l}$ into Eq. (11), we know that $x_{k}^{n^{*},(i)} \sim p\left(x_{k}^{n} x_{k-1}^{n(i)}\right)$.

(4) Update the measurement of Particle filter:

From Eq. (14), we get $Z_{k}^{n}$. Then calculate $q_{k}^{i^{*}}=p\left(z_{k}^{n} \mid x_{k}^{n^{n},(i)}\right)$, and update the weight $q_{k}^{i}=q_{k-1}^{i} q_{k}^{i}$. At last, normalize ${ }_{q_{k}=q_{k}^{*}} / \sum_{i}^{\infty} q^{*}$

(5) Particle filter resampling

Calculate the number of efficient samples $N_{e f f}$. If $N_{\text {eff }}<N_{T}$, use the method of II to resample the particles, and get $\left\{x_{k}^{n, i(i)}, q_{k}^{i}\right\}$.

(6) Update the time of Kalman filter

Calculate $x_{k}^{n}=\sum_{k=1}^{n} q_{k}^{i} n_{k}^{n(i)}$ by $\left\{x_{k}^{n,(i)}, q_{k}^{\prime}\right\}$. Bringing it to $F_{k}^{\prime}\left(x_{k}^{n}\right)=M\left(1-C_{p}^{n}\right) C_{b}^{p} \hat{f}^{b}$, we can find $F_{k}^{l}$, and $x_{k+1 \mid k}^{\prime}=A_{k}^{\prime} x_{k}^{\prime}+F_{k}^{\prime}, \quad P_{k+1 \mid k}^{\prime}=A_{k}^{\prime} P_{k}\left(A_{k}^{\prime}\right)^{T}+Q_{k}^{\prime} \cdot$

(7) Let $\mathrm{k}=\mathrm{k}+1$, and return to (2).

\section{SIMULATIONS}

The dimensional state vector of the system is

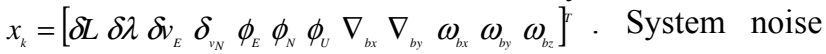
and measurement noise are all Gaussian white noise. The latitude of initial position is $32^{\circ}$, and the longitude is $118^{\circ}$. $\dot{\nabla}_{b}=0$. The constant drift of the accelerometer is $3 \times 10^{3} \mathrm{~g}$. The white noise is $3 \times 10^{3} \mathrm{~g}$. $\dot{\omega}_{\mathrm{b}}=0$. The constant drift of the gyroscope is $0.3^{\circ} / \mathrm{h}$, and the white noise is $0.1 \%$ h. The initial azimuth misalignment angle is $10^{\circ}$, and the initial horizontal misalignment angle is $0.1^{\circ}$. The mean square error of position measurement noise is $3 \mathrm{~m}$. The mean square error of velocity measurement noise is $0.03 \mathrm{~m} / \mathrm{s}$. Under dynamic environment, the carrier is in uniform velocity rectilinear motion, and its speed is $20 \mathrm{~m} / \mathrm{s}$, azimuth angle is $45^{\circ}$, the time of simulation is $800 \mathrm{~m}$. We implement $\mathrm{PF}$ and $\mathrm{K} / \mathrm{PF}$ separately to simulate. Fig1 to Fig7 display the error curve of $\mathrm{PF}$ and $\mathrm{K} / \mathrm{PF}$.

From Fig1, Fig2 and Fig3, it shows that, in the circumstances of the large misalignment angle, $\mathrm{PF}$ and $\mathrm{K} / \mathrm{PF}$ are similar in the convergence rates of course and attitude error are the same. However, the PF is more accurate than $\mathrm{K} / \mathrm{PF}$ in the estimation of course angle and attitude angle. The velocity error and position error can be measured directly, and the two methods are the same in estimation accuracy.

The state dimensions of $\mathrm{PF}$ is 12 , it is 3 for $\mathrm{K} / \mathrm{PF}$. The numbers of particle are 10000 and 600 separately. It takes $389.27 \mathrm{~s}$ to iterative 800 times for $\mathrm{PF}$, and it is $5.07 \mathrm{~s}$ for
$\mathrm{K} / \mathrm{PF}$. From the analysis above, the time the PF consumes is 75.8 times more than the K/PF consumes. From these simulation results, it can be seen that, the K/PF are better than PF in the adaptability, the accuracy and the time.

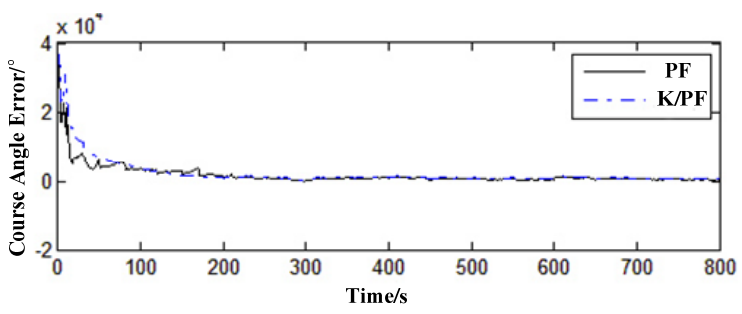

Figure 1. Course angle error

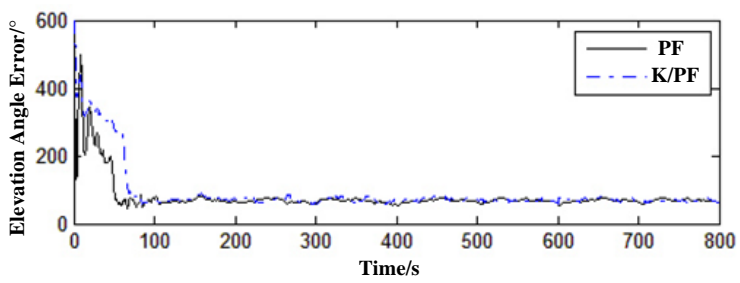

Figure 2. Elevation angle error

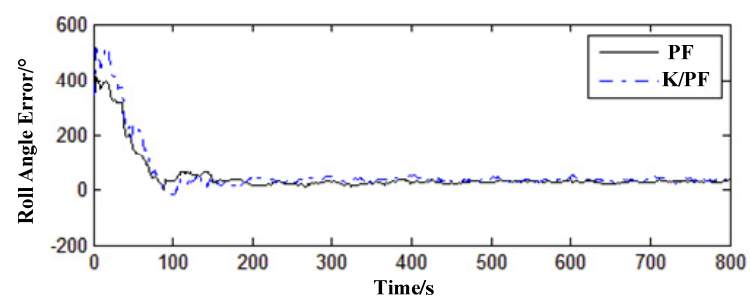

Figure 3. Roll angle error

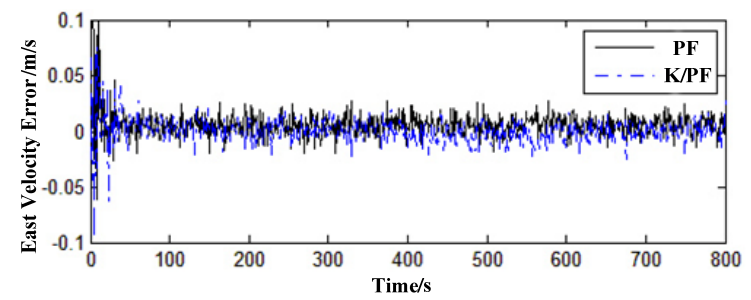

Figure 4. East velocity error

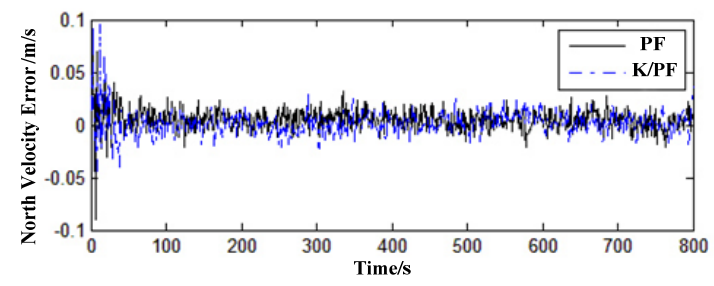

Figure 5. North velocity error 


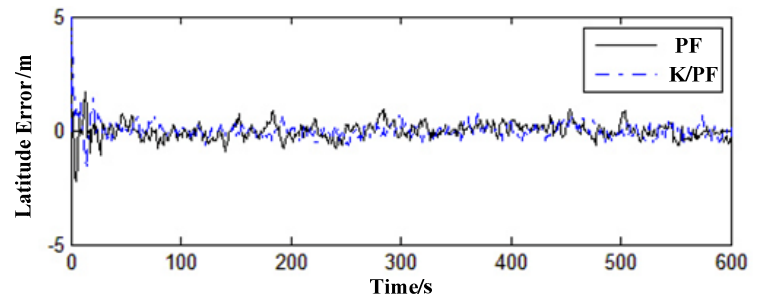

Figure 6. Latitude error

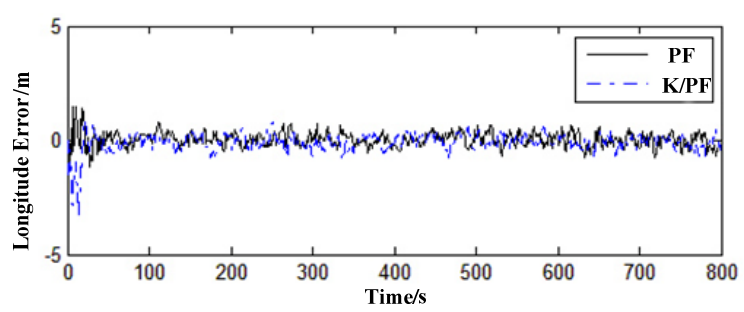

Figure 7. Longitude error

\section{CONCLUSION}

Particle filter is introduced. According to the RaoBlackwellization theorem and the dynastic error model, a new Kalman/Particle mixed filter used on SINS/GPS integrated navigation system was proposed. The new method divides the system into two sub-models, one is linear, the other one is nonlinear, and then implement Kalman filter and particle filter separately. From the simulation results, it can be seen that, the $\mathrm{K} / \mathrm{PF}$ are better in the adaptability, the accuracy and the time.

\section{ACKNOWLEDGMENT}

First and foremost, I would like to show my deepest gratitude to my wife, Dr Zhang Rong. She provided me with valuable guidance in every stage of the writing of this paper.

I shall extend my thanks to Mr. Yang for all his kindness and help.

Last but not least, I'd like to thank all my friends, especially my three lovely workmates, for their encouragement and support.

\section{REFERENCES}

[1] N.J. Gorden, D.J. Salmond, A. Smith, "A novel approach to nonlinear/non-Gaussian Bayesian state estimation," Proc. IEEE Symp. Proceedings on Radar and Signal Processing, IEEE Press, Feb. 1993, pp. 57-60.

[2] M.Y. Fu, Z.H. Deng, J.W. Zhang, The Kalman Filtering Theory and Application in Navigation System, CA: Science Publishing Company, 2003.

[3] Y.X. Xiao, Research on Alignment of Inertial System with Moving Base and Integrated Navigation System, CA: Aviation Aerospace University in Peking, 2002.

[4] M. Sanjeev, S. Maskell, N. Gordon, T. Clapp, "A tutorial on particle filters for online nonlinear/non-Gaussian Bayesian tracking," Proc. IEEE Symp. Transactions on signal processing, IEEE Press, Feb. 2002, pp. 288-295.

[5] K.W. Li, H.L. Wang, "The applied research of federated Kalman filter on fault-tolerant integrated navigation system," Microcomputer Information, vol 22, pp. 119-221, 2006 . 